VPSim: Variance Propagation by Simulation

Tom Burr

C. Alton Coulter

Joan Prommel 


\section{DISCLAIMER}

This report was prepared as an account of work sponsored by an agency of the United States Government. Neither the United States Government nor any agency thereof, nor any of their employees, make any warranty, express or implied, or assumes any legal liability or responsibility for the accuracy, completeness, or usefulness of any information, apparatus, product, or process disclosed, or represents that its use would not infiringe privately owned rights. Reference herein to any specific commercial product, process, or service by trade name, trademark, manufacturer, or otherwise does not necescarily constitute or imply its endorsement, recommendation, or favoring by the United States Government or any agency thereof. The views and opinions of authors expresced herein do not necessarily state or rellect those of the United States Government or any agency thereof. 


\section{DISCLAIMER}

Portions of this document may be illegible electronic image products. Images are produced from the best available original document. 


\title{
VPSim: Variance Propagation by Simulation
}

\author{
by \\ Tom Burr, C. Alton Coulter, and Joan Prommel
}

\begin{abstract}
One of the fundamental concepts in a materials control and accountability system for nuclear safeguards is the materials balance (MB). All transfers into and out of a material balance area are measured, as are the beginning and ending inventories. The resulting MB measures the material loss, $M B=T_{\text {in }}+I_{B}-T_{\text {out }}-I_{E}$. To interpret the $\mathrm{MB}$, we must estimate its measurement error standard deviation, $\sigma_{\mathrm{MB}}$. When feasible, we use a method usually known as propagation of variance (POV) to estimate $\sigma_{\mathrm{MB}}$. The application of POV for estimating the measurement error variance of an $\mathrm{MB}$ is straightforward but tedious. By applying POV to individual measurement error standard deviations we can estimate $\sigma_{\mathrm{MB}}$ (or more generally, we can estimate the variance-covariance matrix, $\Sigma$, of a sequence of MBs). This report describes a new computer program (VPSim) that uses simulation to estimate the $\Sigma$ matrix of a sequence of MBs. Given the proper input data, VPSim calculates the MB and $\sigma_{\mathrm{MB}}$, or calculates a sequence of $n$ MBs and the associated $n$-by- $n$ covariance matrix, $\Sigma$. The covariance matrix, $\Sigma$, contains the variance of each $\mathrm{MB}$ in the diagonal entries and the covariance between pairs of MBs in the off-diagonal entries.
\end{abstract}

\section{Introduction and Outline}

This report describes a computer program (VPSim) that uses simulation to estimate the measurement error variance, $\sigma^{2}$, of a material balance (MB) in nuclear materials accounting. It serves as both a user's manual (as a supplement to VPSim's on-line help) and a final report for the project that funded its development. Given the proper input data, VPSim calculates the MB and its $\sigma^{2}$, or calculates a sequence of $n$ MBs and the associated $n$-by- $n$ measurement error covariance matrix, $\Sigma$. The covariance matrix, $\Sigma$, contains the variance of each MB in the 
diagonal entries and the covariance between pairs of MBs in the off-diagonal entries. The simplest use of $\sigma$ is to assume that $\mathrm{MB} / \sigma$ is approximately normally distributed with mean 0 and variance 1 if there is no loss of material, so that a typical test for material loss would check whether $\mathrm{MB} / \sigma$ exceeded 2 or 3.

The report is organized as follows. Section 2 provides a historical background. Section 3 describes the measurement error models assumed by VPSim, and Section 4 describes how VPSim estimates $\sigma^{2}$. In Section 5 we provide three examples and compare their results to results from another program (Materials Accounting with Sequential Testing, MAWST, used by some Department of Energy sites). Section 5 also gives example input files for VPSim and is the user's manual portion of the report. An online help file is available with the installation disk, so the user's manual is brief. Section 6 is a summary and suggestions for the next version of VPSim.

\section{Background}

One of the fundamental concepts in a materials control and accountability system for nuclear safeguards is the materials balance. All transfers into and out of a material balance area (MBA) are measured, as are the beginning and ending inventories. The resulting materials balance measures the material loss, $M B=T_{\text {in }}+I_{B}-T_{\text {out }}-I_{E}$. The application of propagation of variance (POV) for estimating the variance of an $\mathrm{MB}$ is straightforward but tedious (see Refs. 2 and 3). The main application of POV in safeguards is to use individual measurement error standard deviations to estimate the measurement error standard deviation of an $\mathrm{MB}, \sigma_{\mathrm{MB}}$ (or more generally, to estimate the variance-covariance matrix, $\Sigma$, of a sequence of MBs). Because many terms are summed in the MB equation, the central limit theorem (CLT) ${ }^{1}$ can be applied to indicate that an MB should be approximately normally distributed with mean equal to the true loss and with standard deviation $\sigma_{\mathrm{MB}}$. As an aside, some of the terms summed in a typical MB calculation will have nonzero covariance under typical measurement error models because they are made by the same instrument during the same calibration period. Currently, this is the only source of nonzero covariance that VPSim error models recognize, and it is commonly referred to as instrument bias or systematic error. There are many versions of the CLT, some of which allow the terms to have nonzero covariance. However, with our interpretation of the biases as "fixed during the calibration period" but otherwise random, the ordinary CLT for independent terms applies, and we simply must account for the nonzero covariances when we estimate $\sigma_{\mathrm{MB}}$. 
As a simple example of POV, suppose we have measurement error models for both volume $\mathrm{V}$ and concentration $\mathrm{C}$ of uranium $\mathrm{U}$ and that the error models include estimates of the uncertainty (total error standard deviation). The mass $\mathrm{M}$ of $\mathrm{U}$ would be estimated by $\mathrm{M}=\mathrm{VC}$, and via POV we have $\sigma_{M}^{2}=V^{2} \sigma_{C}^{2}+C^{2} \sigma_{V}^{2}$ (approx.), where $\sigma_{C}^{2}$ is the total concentration measurement error variance ("systematic" plus "random" in typical error models), and similarly for $\sigma_{\mathrm{V}}^{2}$. Usually, the application of POV to estimate $\sigma_{\mathrm{MB}}$ is straightforward but tedious. Because of this tedious nature, there are several software tools in the domestic and international safeguards communities to help implement POV. Each tool has its own strengths and weaknesses. This paper describes a new software tool (VPSim) whose main strength is that its required input data files are relatively simple to create. The measurement error process is simulated using simple Monte Carlo simulation and the individual measurements such as $\mathrm{V}$ and $\mathrm{C}$ above are combined using a calculator-type evaluation.

Because of the tedious nature of POV, several computer codes exist today to help perform POV. Examples include MAWST ${ }^{2}$ and Variance Propagation (VP) used for training. Also, some sites have such simple error models that custom "spreadsheet-like" calculations are adequate. Any software used to perform POV will have its strengths and weaknesses. A main disadvantage of MAWST is probably its limited form of error models. This limited form forces the user to use cryptic pseudo-measurements to effectively extend the allowed error models. A common example is to include sampling error in the total random error by dividing the actual measurement into two pseudo-measurements.

Because the application of POV can be tedious and input files can be presented in multiple ways to MAWST, it is valuable to have an alternative method to compare results. This report describes a new code, VPSim, that uses Monte Carlo simulation to do POV. VPSim does not need to rely on pseudo-measurements. It is written in $\mathrm{C}++$, runs under Windows $\mathrm{NT}$, and has a user-friendly interface. VPSim has been tested on several example problems, and in this report we compare its results to results from MAWST for three example problems. We also describe its error models and indicate the structure of its input files. A main disadvantage of VPSim is its long run times. If many simulations are required $(20,000$ or more, repeated two or more times) and if each balance period has many (10,000 or more) measurements, then run times an can be an hour or more. For small- and modest-sized problems, run times are a few minutes. The main advantage of VPSim is that its input files are simple to construct and, therefore, also are relatively easy to inspect. 


\section{VPSim's Error Models}

The two common error models in safeguards are the multiplicative and additive models. ${ }^{2,3}$ A multiplicative error model for a measured volume $V_{m}$ as a function of true volume $V_{T}$ is $\mathrm{V}_{\mathrm{m}}=\mathrm{V}_{\mathrm{T}}+\mathrm{V}_{\mathrm{T}} \mathrm{S}+\mathrm{V}_{\mathrm{T}} \mathrm{R}$, where $\mathrm{S}$ is a systematic error and $\mathrm{R}$ is a random error. The model is fully specified by specifying the systematic and random error standard deviations $\sigma_{S}$ and $\sigma_{R}$ (and assuming $\mathrm{S}$ and $\mathrm{R}$ each have a normal [Gaussian] distribution with zero mean). An additive model for $\mathrm{V}$ is $\mathrm{V}_{\mathrm{m}}=\mathrm{V}_{\mathrm{T}}+\mathrm{S}+\mathrm{R}$ and again $\mathrm{S} \sim \mathrm{N}\left(0, \sigma_{\mathrm{S}}{ }^{2}\right)$ and $\mathrm{R} \sim \mathrm{N}\left(0, \sigma_{\mathrm{R}}{ }^{2}\right)$, where $\mathrm{N}\left(0, \sigma_{\mathrm{S}}{ }^{2}\right)$ denotes the normal distribution with mean zero and variance $\sigma_{\mathrm{S}}{ }^{2}$. Not all error models are either multiplicative or additive, nor must there be only one random error source or one systematic error source for a given measurement. Therefore, VPSim uses the more general error model,

$$
\mathrm{V}_{\mathrm{m}}=\mathrm{V}_{\mathrm{T}}+\mathrm{V}_{\mathrm{T}}^{\alpha \mathrm{S} 1} \mathrm{~S}_{1}+\mathrm{V}_{\mathrm{T}}^{\alpha \mathrm{S} 2} \mathrm{~S} 2+\ldots+\mathrm{V}_{\mathrm{T}}^{\alpha \mathrm{R} 1} \mathrm{R}_{\mathrm{I}}+\mathrm{V}_{\mathrm{T}}{ }^{\mathrm{aR} 2} \mathrm{R}_{2+}+\ldots
$$

where $\alpha_{\mathrm{Sl}}$ is a coefficient for the first systematic error that determines how $V_{T}$ affects the standard deviation $\left(\alpha_{s 1}=1\right.$ corresponds to a multiplicative error model and $\alpha_{s 1}=0$ corresponds to an additive error model). This error model easily accommodates models with both short- and long-term systematic errors, and models that separate the random analytical chemistry error from the random sampling error. If used in a straightforward way, MAWST allows only 0 or 1 systematic and random errors, and both must be either multiplicative or additive. MAWST "tricks" such as pseudo-measurements have been developed to get around this limitation.

A VPSim error model such as Eq. (1) is fully specified by supplying the $\alpha$ 's and the $\sigma_{\mathrm{S}}$ and $\sigma_{\mathrm{R}}$ for all errors. We expect that calibration and/or measurement control data is used to estimate the $\alpha$ 's and all of the $\sigma_{\mathrm{S}}$ and $\sigma_{\mathrm{R}}$. We also realize that there are difficulties with interpretation of many MBs. Two main difficulties are (1) holdup (unmeasured inventory) and (2) incorrect measurement error models (often resulting because measurement performance on standards is better than on real items). Our interest here is strictly in making POV as simple as possible to perform.

\section{VPSim's Approach to POV}

The user supplies an input file that specifies how to combine individual measurements. For example, if a level reading $\mathrm{L}$ is the "raw" data, which is converted to volume $\mathrm{V}$ via $\mathrm{V}=\mathrm{a}+\mathrm{bL}$, which is then multiplied by $\mathrm{C}$, the user specifies that the mass of interest is obtained by the formula (meas[0] + meas[1]* meas[2]) * meas[3]. Note that the estimated intercept $a$ and the estimated slope $b$ are treated as measurements (whose error models could be deduced from calibration data), and note that each assay involves the four measured values: a, 
b, $\mathrm{L}$, and $\mathrm{C}$. To simulate the measurement error process for a given measured value, a new random error is drawn from an $N\left(0, \sigma_{R}^{2}\right)$ distribution with the appropriate value of $\sigma_{\mathrm{R}}^{2}$. The systematic errors require careful bookkeeping. For a given simulation, all measurements made by the same instrument during the same calibration period are assumed to share the same systematic error, so only the first measurement made by a given instrument during a given calibration period has a "new" systematic error. This "new" systematic error is randomly drawn from an $\mathrm{N}\left(0, \sigma_{s}^{2}\right)$ distribution. All subsequent measurements by a given instrument during a given calibration period share that same systematic error. The user specifies which MB and balance period a given measured value is for, and whether that measured value is $T_{\text {in }}$ (an input transfer), $T_{\text {out }}$ (an output transfer), $I_{B}$ (a contribution to beginning inventory), or $I_{E}$ (a contribution to ending inventory) in the MB. If the number of balance periods is 12 and the number of simulations nsim $=1000$, then VPSim computes

$$
\begin{array}{clll}
\mathrm{MB}_{1,1}, & \mathrm{MB}_{1,2}, & \ldots, & \mathrm{MB}_{1,12} \\
\mathrm{MB}_{2,1}, & \mathrm{MB}_{2,2}, & \ldots, & \mathrm{MB}_{2,12} \\
\vdots & & & \\
\mathrm{MB}_{1000,1}, & \mathrm{MB}_{1000,2}, \ldots, & \mathrm{MB}_{1000,12}
\end{array}
$$

The 12-by-12 variance-covariance matrix $\Sigma$ (with variances of each $M B$ on the diagonal, covariances between pairs of MBs on the off-diagonals) of this 1000-by-12 matrix of MBs is the estimated $\Sigma$ of the actual MB sequence. The user can repeat the calculation with a second set of 1000 simulations to see if there is sufficiently close agreement in the two estimated $\Sigma$ s to suggest that 1000 was enough simulations.

\section{Examples}

We will present three examples and compare VPSim results to results from MAWST for each. The input transfers, output transfers, beginning inventory, and ending inventory are denoted as TI, TO, BI, and EI, respectively. If an entry is the EI for a given balance period, then it automatically is ASSUMED to be part of BI for the next balance period, so it should not be explicitly included as part of $\mathrm{BI}$ for the next balance period.

\subsection{Example 1}

We consider a small example (from Section 7 in Ref. 2) with two measurement types: (1) an NDA measurement (nondestructive assay, which estimates the mass $M$ of $U$ ) and (2) a weight times a concentration measurement (DA, destructive chemical assay with sampling error) to estimate M. Figure 1 shows the three required input files (ex1.000, ex1.err, and ex1.cmb) and one output file (ex1.out). The output file contains the sequence of MBs and the 
estimated $\Sigma$ for the sequence of MBs. For this test case, estimated $\Sigma$ using VPSim is the same (within measurement error) as the estimated $\Sigma$ using MAWST.

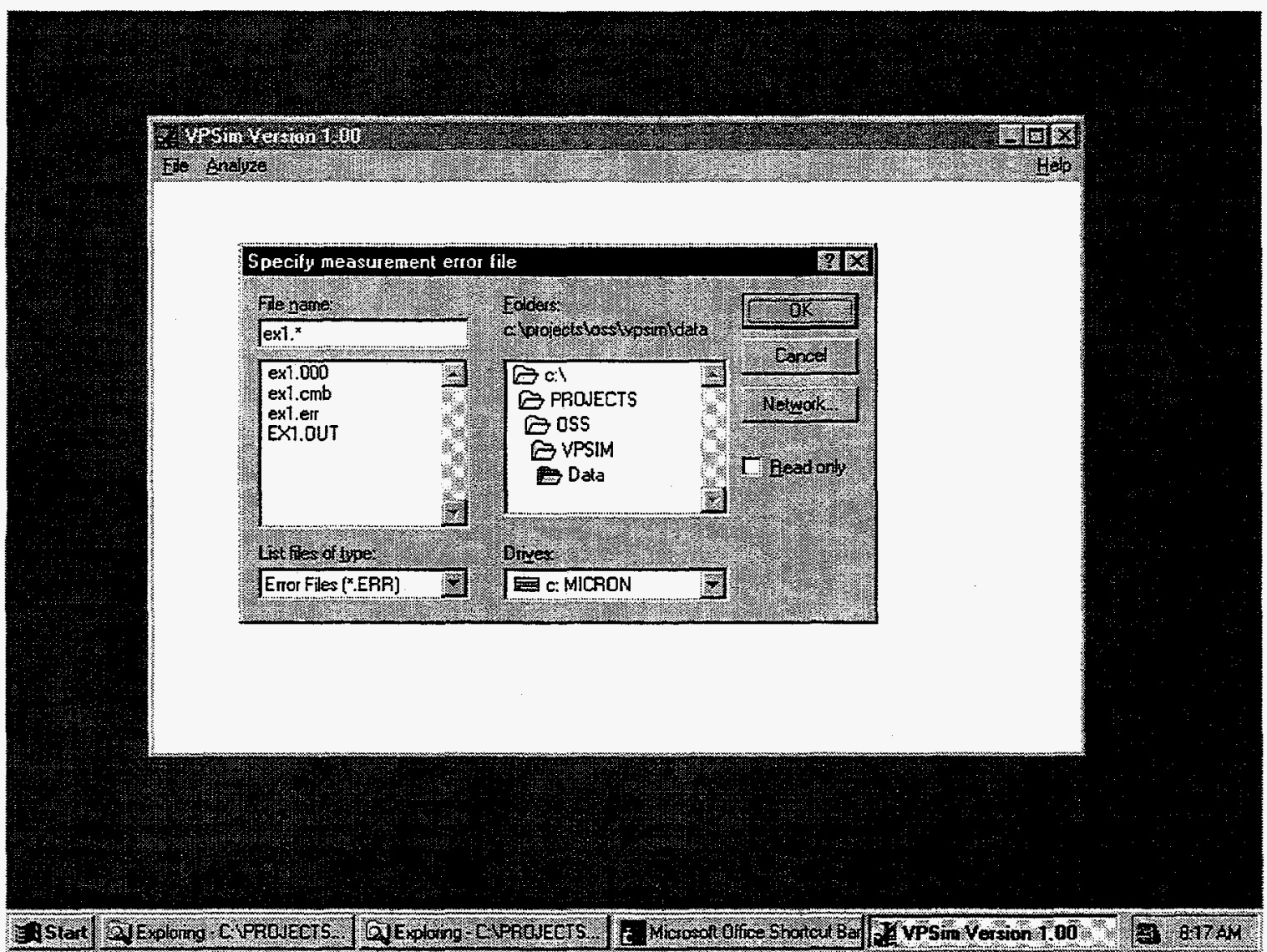

Figure 1. Three VPSim input files and one output file.

The measurement data is in ex1.000. The measurement error standard deviations are in ex1.err (error file). The algebra needed to combine measurements (such as $(\operatorname{meas}[0]+\operatorname{meas}[1] *$ meas[2]) $*$ meas[3]) is in ex1.cmb (combinations file).

The ex1.cmb file contains

TYPE1 meas[0] and

TYPE2 (meas[0]-meas[1])*meas[2].

The TYPE1 measurement is the NDA measurement and the TYPE2 measurement is (full container weight minus empty container weight) times concentration.

The ex1.err file contains:

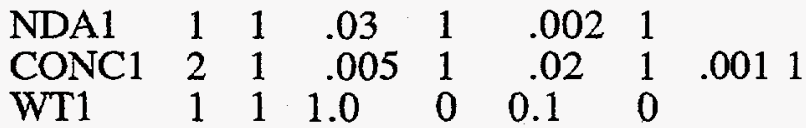

For the NDA1 measurement, the $\begin{array}{llllll}1 & 1 & .03 & 1 & .002 & 1 \text { row indicates that there is one }\end{array}$ random error and one systematic error. For the random error, $\sigma_{R}=3 \%$ (with $\alpha_{R}=1$, so it is 
a multiplicative error). For the systematic error, $\sigma_{\mathrm{s}}=0.2 \%$ (with $\alpha_{\mathrm{s}}=1$, so it is a multiplicative error). For the $\mathrm{CONC1}$ measurement, there are two random errors (one for analytical and one for sampling) and there is one systematic error.

The ex1.000 file contains, in part (the complete input file for MAWST is available with the MAWST distribution disk, and could be used to construct the entire ex1.000 file).

$\begin{array}{clll}\text { TYPE1 } & & & \\ 1 & 1 & \text { BI } & \\ 52.62 & \text { NDA1 } & 1 & 1 \\ 1 & 1 & \text { EI } & \\ 48.09 & \text { NDA1 } & 2 & 2 \\ \text { TYPE2 } & & & \\ 1 & 1 & \text { BI } & \\ 120 & \text { WT1 } & 1 & 1 \\ 25 & \text { WT1 } & 1 & 2 \\ .484 & \text { CONC1 } & 1 & 1 \\ \text { TYPE1 } & & & \\ 1 & 2 & \text { TO } & \\ 48.09 & \text { NDA1 } & 2 & 2\end{array}$

Note that the measured values are grouped by measurement type, but each measurement type may appear multiple times in the file. For the first measurement (52.62 $\mathrm{g}$ by NDA1), the "1 1 BI" row indicates MBA1, balance period 1, and Beginning Inventory; and the "52.62 NDA1 11 " row indicates the measured value, the instrument ID (unique ID), the calibration period for the first (of one here) systematic error, and a time stamp for that calibration period. We believe that this file is simple to construct and interpret.

In Ex1.out we show the results of running this small problem for two sets of 10,000 simulations (run time is approximately 1 second for 10,000 simulations of this small problem).

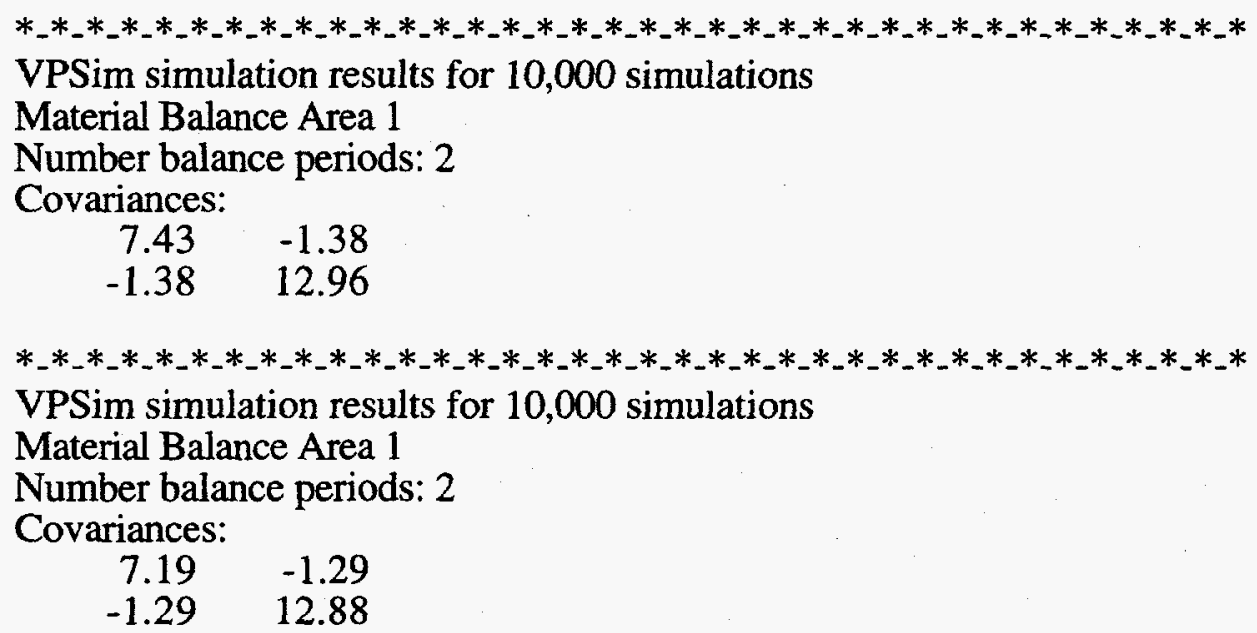


The user can compare the two estimates of $\Sigma$ and then decide if more simulations are needed. In this case, we see that it would be good to increase the number of simulations to perhaps 20,000, mostly because the estimate of $\sigma_{12}^{2}$ (the covariance between $\mathrm{MB}_{1}$ and $\mathrm{MB}_{2}$ ) varies from -1.4 to -1.3 in the two simulations.

The MAWST result for $\Sigma$ is

Covariances:

7.28

$-1.34 \quad 12.94$

The MAWST result is approximate because it keeps only the first (linear) terms in a Taylor series approximation to the assumed algebraic form (sum of products of sum of products). The VPSim result is also approximate because it is always based on a finite number of simulations.

\subsection{Example 2}

This example for two balance periods comes from the Elektrostal Fuel Fabrication Facility near Moscow, Russia. We have simplified it to consider only inputs and outputs. There are 10 inputs and 1 output for both balance periods. The only measurements are weights of powder containing uranium and concentrations of uranium in the powder. We assume that all measurements are recalibrated after the first balance period. The example $2 . \mathrm{cmb}$ file contains

TYPE1 (meas[0]-meas[1])*meas[2]

The example2.001 file contains for balance period 1, in part,

TYPE1

$\begin{array}{clll}1 & 1 & \text { TI } \\ 5500 & \text { wt1 } & 1 & 1 \\ 500 & \text { wt1 } & 1 & 2 \\ .02 & \text { conc1 } & 1 & 1 \\ 1 & 1 & \text { TI } & \\ 5500 & \text { wt1 } & 1 & 3 \\ 500 & \text { wt1 } & 1 & 4 \\ .02 & \text { conc1 } & 1 & 2 \\ \vdots \text { (part of file omitted here) } & \\ 1 & 1 & \text { TO } & \\ 55100 & \text { wt2 } & 1 & 1 \\ 5000 & \text { wt2 } & 1 & 2 \\ .02 & \text { conc2 } & 1 & 1\end{array}$

And the second balance period input is the same except the calibration period changes from 1 to 2 for each measurement. 
In Ex2.out we show the results of running this small problem for two sets of 20,000 simulations (run time is approximately 10 seconds for 1000 simulations of this small problem).

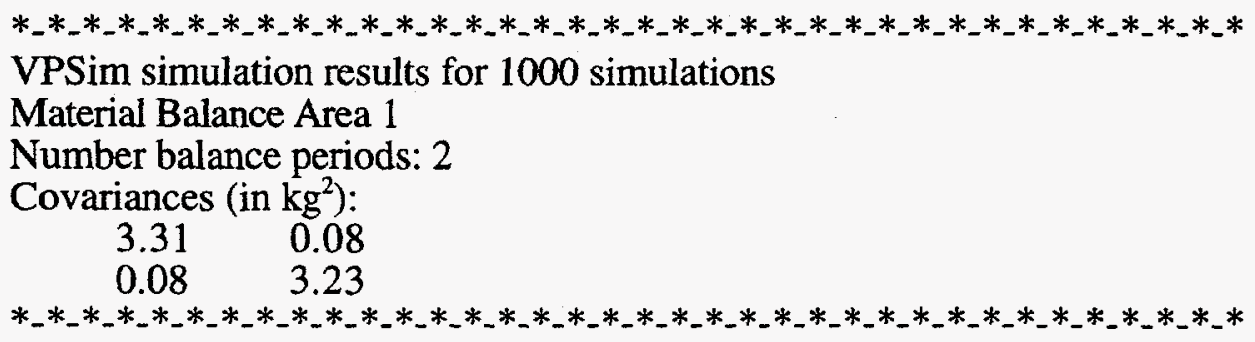

VPSim simulation results for 1000 simulations

Material Balance Area 1

Number balance periods: 2

Covariances:
3.39
0.00
$0.00 \quad 3.25$

The MAWST result for $\Sigma$ is

Covariances:
3.25
0.0
0.0
3.25

In this case, 1000 simulations gives adequate repeatability and reasonable agreement with the MAWST result. When we use 20,000 simulations, repeatability and agreement with MAWST is excellent.

\subsection{Example 3}

This example is typical of what we expect at a reprocessing plant main chemical processing area where there are many large tanks holding plutonium solution. To simplify the presentation, only transfers are considered. There are 22 inputs into tank 1 and 5 outputs from tank 15 during balance period 1 , and 23 inputs, 6 outputs during balance period 2 .

The example3.cmb file contains

TYPE1 meas[0] (not used here, but could be used to estimate in process inventory) TYPE2 meas[0]*meas[1] (volume times plutonium concentration) 
The example3.001 file contains, in part

TYPE2

$\begin{array}{clll}1 & 1 & \text { TI } & \\ 20000 & \text { volumel } & 1 & 1 \\ 2.76 & \text { conc1 } & 1 & 1 \\ 1 & 1 & \text { TI } & \\ 20000 & \text { volume1 } & 1 & 2 \\ 2.76 & \text { conc1 } & 2 & 1\end{array}$

$\begin{array}{clll}1 & 1 & \text { TO } & \\ 830 & \text { volume 15 } & 1 & 1 \\ 254.94 & \text { conc15 } & 1 & 1 \\ 1 & 1 & \text { TO } & \\ 830 & \text { volume15 } & 1 & 2 \\ 254.94 & \text { conc15 } & 2 & 1\end{array}$

These two balance periods each have a throughput of approximately $1200 \mathrm{~kg}$ of plutonium.

In Ex3.out we show the results of running this small problem for two sets of 20,000 simulations (run time is approximately 20 seconds for 20,000 simulations of this small problem).

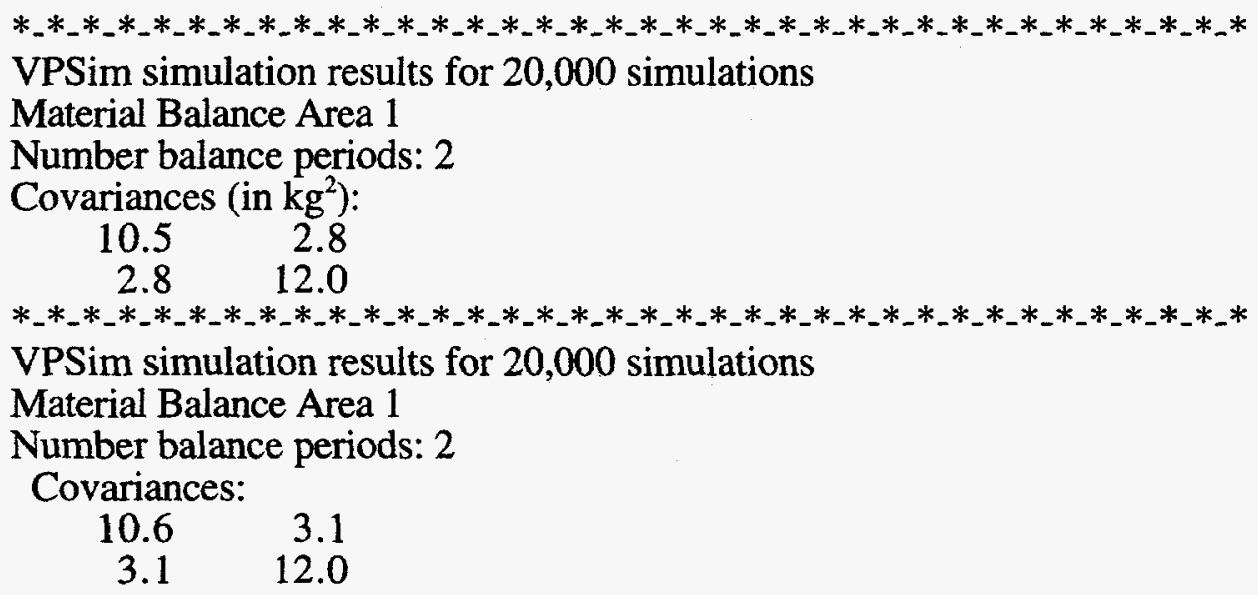

The MAWST result for $\Sigma$ is

Covariances:

$\begin{array}{rr}10.4 & 2.9 \\ 2.9 & 12.0\end{array}$

Again, the VPSim results agree closely with the MAWST results for $\Sigma$. 


\section{Summary, Conclusions, and Recommendations}

The Department of Energy facilities now have three main computer codes to perform variance propagation:

1. VP uses "forms" like $M=V C, M=W C$, etc., and uses "stream averages." VP is very useful for "system studies," but is not as useful for actual operations where there can be considerable fluctuation about stream averages.

2. MAWST uses no "forms," but must express any material balance as a sum of products of a sum of products. Analytical variance propagation for that form was done by hand, then coded into MAWST.

3. VPSim is now another option. VPSim uses no "forms." It estimates $\Sigma$ for a sequence of MBs by simulation. We have tested VPSim results on three examples (the one presented in Section 3 and two others). We tested the results by doing hand calculations and by running MAWST on the same examples. Our VPSim, hand-calculations, and MAWST results all agree within measurement error. There could be pathological cases where we actually prefer the VPSim results to the MAWST or hand-calculation results because of the approximations used in MAWST or in hand calculations. We are not aware of any real cases where the MAWST-type approximations are inadequate, however.

A small shortcoming of version 1.0 of VPSim is that all measurement error models are assumed to be Gaussian. It would be simple to extend the choices to include other common models such as uniform and log-normal. It would also be simple to then assess the assumption that the MB sequence is approximately multivariate normal (by appeal to the central limit theorem) in distribution. We consider this level of error model scrutiny to be beyond what is typically needed. Also, in the early 1980s there was an effort to include MAWST error models in a simulation code (MASIM, written by Andy White at Argonne National Laboratory), so VPSim is to our knowledge the second simulation-based approach to POV for estimating the $\Sigma$ of an MB sequence. MASIM was written in Fortran77 to run on a VAX. It did not extend the MAWST error models the way VPSim does, and most importantly it did not simplify the input data files; it used the same input files that MAWST does. Our main two motives for writing VPSim were (1) to simplify the input data files and (2) to provide an alternative tool for doing POV so results could be compared.

MAWST has an attractive feature (that relies on the approximate analytical expressions for POV) for ranking the sources of variance in $\Sigma$. A "methods" file can be specified and the contribution to $\Sigma$ by each method is given. For example, all volume measurements could be method 1 and all concentration measurements could be method 2 . To do the same thing in VPSim currently requires that the user manually set the volume measurement errors to zero, 
run the simulations, and note the change in $\Sigma$, then repeat for the concentration measurements. A desirable added feature would be to automate this process using a methods file, as does MAWST. Because the simulations have to be repeated for each method (setting all error variances to zero in the method), the total run times could be on the order of 1 hour for typicalsized problems.

Another desirable added feature would be to generalize the calculations to include shipper-receiver differences, the " $D$ " statistic used by the International Atomic Energy Agency ( $\mathrm{D}$ is the estimated difference between the operator and inspector $\mathrm{MB}$ ), and other desired calculations in addition to the current MB calculations. This would be a minor generalization that would require the input files to communicate which terms are added and which are subtracted to calculate, for example, the $\mathrm{D}$ statistic. There would also be a bookkeeping change that involves how VPSim currently assumes that the EI for a given balance period is the BI for the next period.

VPSim is a new tool for doing POV. Version 1.0 has on-line help, is distributed via an installation disk, and is available for use under Windows NT. The usual caveats apply: if the individual measurement uncertainties are poorly estimated (often understated) or if fluctuating holdup is ignored, then the POV-based estimates of $\sigma_{\mathrm{MB}}$ or $\Sigma_{\mathrm{MB}}$ are difficult to interpret. For facilities that are ready to apply POV, we are happy to offer both MAWST and VPSim for consideration. We believe that VPSim will be easier to use but we would consider it valuable to compare the MAWST results to VPSim results. We welcome user response on the feasibility of VPSim for large data sets (large is 1000 or more measurements for each of 12 or more balance periods) and announce the availability of the software in Ref. 4.

\section{References}

1. M. Degroot, Probability and Statistics (Addison-Wesley Publishing Company, Reading, Massachusetts, 1986).

2. R. Picard and J. Hafer, "MAWST: Materials Accounting With Sequential Testing," Los Alamos National Laboratory, Safeguards Systems Group report N-4/89-111 (1989).

3. W. Bowen and C. Bennett, eds., "Statistical Methods for Nuclear Materials Management," Nuclear Regulatory Commission report NUREG/CR-4604 (1988).

4. T. Burr, C. Alton Coulter, and J. Prommel, "Variance Propagation by Simulation," presented at the 38th Annual Meeting of the Institute of Nuclear Materials Management, Phoenix, Arizona, July 20-24, 1997 (to be published in proceedings); Los Alamos National Laboratory document LA-UR-97-2549. 